\title{
Wind Turbine Modeling Based on A Multi-model LPV Structure
}

\author{
Liren $\mathrm{Cao}^{1}$, Xiaorong $\mathrm{Hu}^{1}$, Ruohan $\mathrm{Chen}^{1}$ and Guoli $\mathrm{Ji}^{1,{ }^{*}}$ \\ ${ }^{1}$ Department of Automation, Xiamen University, Xiamen, China \\ *Corresponding author: glji@xmu.edu.cn
}

Keywords: Multi-model LPV model; Wind turbine; OE models; Linear functions.

\begin{abstract}
This paper establishes a multi-model linear parameters varying (LPV) model for wind turbine. The multi-model LPV model is composed by several local linear models interpolating with weighting functions. Local linear models are identified at different wind speeds and pitch angles in the form of output error (OE) models. Linear functions are used as weighting functions. A simulated mechanical wind turbine process is employed to test the usefulness of the method.
\end{abstract}

\section{Introduction}

The use of renewable energy is becoming more and more important with the global energy shortage and environmental pollution. Wind energy is one of the most promising renewable energy. The study of wind turbines therefore is significantly increasing the attentions nowadays. It has been shown in [1] that a well-tuned control system has big effect on reducing the cost of wind power generation. In order to improve the wind turbine system performance, it is necessary to have models that describe the dynamics of wind turbine. Mechanistic-based models have been widely used. For example, mechanism models in the form of Simulink were constructed in [2]. This kind of models requires to present the relationship of all factors based on some strict assumptions, which may introduce some difficulties in application.

A multi-model LPV structure was proposed for nonlinear system identification in [3], where linear local models were interpolated by different weighting functions. Ji et al. proposed single scheduling variable multi-model LPV method to establish a circulating fluidized bed boiler model [4]. Double scheduling variable multi-model LPV method was proposed and applied in the identification of high purity distillation column in [5]. Following these ideas, this paper identifies a multi-model LPV model for Wind Turbine.

\section{Wind Turbine Review}

Model Structure of Wind Turbine. A simplified wind turbine system is shown in Fig. 1, which is composed by blade and pitch subsystem, drive train subsystem, generator and converter subsystem. Variables of subsystems are defined as follows: $\beta_{\text {ref }}$ is the reference of the pitch angle, $v$ is the wind speed acting on the turbine blades, $T_{a}$ is the rotor torque, $\omega_{r}$ is the rotational speed of the rotor, $\omega_{g}$ is the rotational speed of the generator, $T_{g}$ is the generator torque, $P_{g}$ is the power produced by the generator. Note that the inputs of system are $v$ and $\beta_{\text {ref }}$. The outputs are $\omega_{g}$ and $P_{g}$.

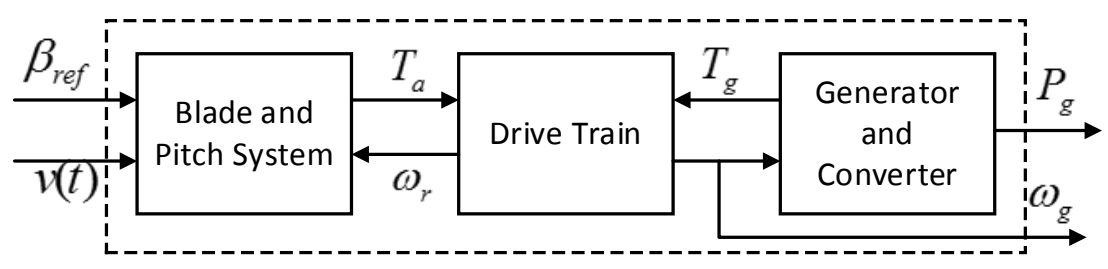

Fig. 1. The structure of wind turbine 
Wind Model. A mathematical model is used to generate a wind speed sequence by Junyent-Ferré et al. [6]. This wind speed expression has the form:

$$
v(t)=v_{m}(t)+v_{t}(t)
$$

where $v_{m}(t)$ is a mean wind speed which contains a constant component and a common ramp component. $v_{t}(t)$ is a turbulence component which is generated by white noise sequence $e(t)$ and a constant $C$ :

$$
v_{t}(t)=\frac{c}{1-0.99 q^{-1}} e(t)
$$

Aerodynamics Model. The aerodynamics of the wind turbine is modeled as the rotor torque $T_{a}$, which is generated by wind. $T_{a}$ can be expressed as Eq. 3.

$$
T_{a}=\frac{1}{2} \rho \pi R^{3} v^{2} C_{q}(\lambda, \beta)
$$

where $R$ is the radius of the blades. $C_{q}(\lambda, \beta)$ is the torque coefficient depending on the tip speed ratio $\lambda$ and the pitch angle $\beta$. $\lambda$ can be expressed as Eq. 4 .

$$
\lambda=\frac{R \omega_{r}}{v}
$$

Pitch System Model. Pitch system is a actuator which changes $\beta_{\text {ref }}$ to $\beta$, The system can be modeled by a transfer function [7]:

$$
\frac{\beta}{\beta_{\text {ref }}}=\frac{\omega_{n}^{2}}{s^{2}+2 \cdot \zeta \cdot \omega_{n}+\omega_{n}^{2}}
$$

Drive Train Model. The drive train includes a gear box to increase the rotational speed from the low-speed rotor side to the high-speed generator side. The drive train function is expressed as Eq. 6 .

$$
\left[\begin{array}{c}
\dot{\omega}_{r} \\
\dot{\omega}_{g}
\end{array}\right]=\left[\begin{array}{cc}
-\frac{D_{s}}{J_{r}} & \frac{D_{s}}{J_{r} N_{g}} \\
\frac{D_{s}}{J_{g} N_{g}} & \frac{-D_{s}}{J_{g} N_{g}^{2}}
\end{array}\right]\left[\begin{array}{l}
\omega_{r} \\
\omega_{g}
\end{array}\right]+\left[\begin{array}{l}
\frac{T_{a}}{J_{r}} \\
-\frac{T_{g}}{J_{g}}
\end{array}\right]+\left[\begin{array}{l}
-\frac{K_{s}}{J_{r}} \\
\frac{K_{s}}{J_{g}}
\end{array}\right] \delta
$$

where $J_{r}$ and $J_{g}$ are the moments of inertia of the low-speed shaft and the high-speed shaft, $D_{s}$ is the torsion damping coefficient, $N_{g}$ is the gear ratio, $K_{s}$ is the torsion stiffness, $\delta$ is the twist of the flexible drive train.

Generator and Converter Model. The power produced by the generator is given by

$$
P_{g}(t)=\eta \omega_{g}(t) T_{g}(t)
$$

where $\eta$ is the efficiency of the generator. $T_{g}$ can be obtained from the generator torque actuator. The reference of $T_{g}$ is given by the square of $\omega_{g}$.

\section{Multi-model LPV Model}

Multi-model LPV model is interpolated by a number of local linear models with weighting functions. Given a multi-input single-output (MISO) system with $m$ inputs $u_{1}(t), \cdots, u_{m}(t)$ and one output $y(t)$ 
at time $t$. $p_{1}(t)$ and $p_{2}(t)$ are two scheduling variables which can be measured or estimated from the input-output data. $n_{1} \times n_{2}$ operating points are selected for scheduling variables:

$$
p_{1}(t): p_{1, \min } \leq p_{1,1}<\cdots<p_{1, n_{1}} \leq p_{1, \max } ; p_{2}(t): p_{2, \min } \leq p_{2,1}<\cdots<p_{2, n_{2}} \leq p_{2, \max } ;
$$

Then the system has $n_{1} \times n_{2}$ local linear models. The global output can be presented by using local model outputs and their weighting functions:

$$
\hat{y}(t)=\sum_{i=1}^{n_{1}} \sum_{j=1}^{n_{2}} \alpha^{i, j}\left[p_{1}(t), p_{2}(t)\right] \cdot \hat{y}^{i, j}(t)
$$

where $\hat{y}^{i, j}(t)$ is the output of local linear model on operating point $\left\{p_{1, i}, p_{2, j}\right\}$. For the local model structure, we use the OE model as Eq. 9.

$$
\hat{y}^{i, j}(t)=\frac{B_{1}^{i, j}\left(q^{-1}\right)}{A_{1}^{i, j}\left(q^{-1}\right)} u_{1}(t)+\cdots+\frac{B_{m}^{i, j}\left(q^{-1}\right)}{A_{m}^{i, j}\left(q^{-1}\right)} u_{m}(t)+e^{i, j}(t)
$$

where $e^{i, j}(t)$ is the white noise. $q^{-1}$ is the unit time delay operator. $A_{k}^{i, j}\left(q^{-1}\right)$ and $B_{k}^{i, j}\left(q^{-1}\right)$ are polynomials.

$$
\begin{aligned}
& A_{k}^{i, j}\left(q^{-1}\right)=1+a_{1}^{i, j, k} q^{-1}+\cdots+a_{r}^{i, j, k} q^{-r} ; \\
& B_{k}^{i, j}\left(q^{-1}\right)=b_{1}^{i, j, k} q^{-1}+\cdots+b_{r}^{i, j, k} q^{-r} ;(k=1, \cdots, m) .
\end{aligned}
$$

$\alpha^{i, j}\left[p_{1}(t), p_{2}(t)\right]$ is the joint weighting function for scheduling variables $p_{1}(t)$ and $p_{2}(t)$, which can be separated as the product of each linear functions [5].

$$
\alpha^{i, j}\left[p_{1}(t), p_{2}(t)\right]=\alpha^{i}\left[p_{1}(t)\right] \cdot \alpha^{j}\left[p_{2}(t)\right]
$$

where

$$
\begin{gathered}
\alpha^{i}\left[p_{1}(t)\right]=\left\{\begin{array}{cc}
\frac{p_{1}(t)-p_{1, i-1}}{p_{1, i}-p_{1, i-1}} & 2 \leq i \leq n_{1} \& p_{1, i-1} \leq p_{1}(t)<p_{1, i} \\
\frac{p_{1, i+1}-p_{1}(t)}{p_{1, i+1}-p_{1, i}} & 1 \leq i \leq n_{1}-1 \& p_{1, i} \leq p_{1}(t)<p_{1, i+1} \\
1 & i=1 \& p_{1}(t)<p_{1,1} \| i=n_{1} \& p_{1}(t) \geq p_{1, n_{1}} \\
0 & \text { others }
\end{array}\right. \\
\alpha^{j}\left[p_{2}(t)\right]=\left\{\begin{array}{cc}
\frac{p_{2}(t)-p_{2, j-1}}{p_{2, j}-p_{2, j-1}} & 2 \leq j \leq n_{2} \& p_{2, j-1} \leq p_{2}(t)<p_{2, j} \\
\frac{p_{2, j+1}-p_{2}(t)}{p_{2, j+1}-p_{2, j}} & 1 \leq j \leq n_{2}-1 \& p_{2, j} \leq p_{2}(t)<p_{2, j+1} \\
1 & j=1 \& p_{2}(t)<p_{2,1} \| j=n_{2} \& p_{2}(t) \geq p_{2, n_{2}} \\
0 & \text { other }
\end{array}\right.
\end{gathered}
$$

\section{Simulations}

A mechanism model of wind turbine [2] is employed to simulate the proposed approach. $v$ and $\beta_{\text {ref }}$ are selected as scheduling variables respectively. The operating points are chosen as:

$$
p_{1,1}=9, p_{1,2}=12, p_{1,3}=15 ; p_{2,1}=3, p_{2,2}=5, p_{2,3}=9 \text {. }
$$


The simulated LPV model outputs are compared to the measured outputs and global linear model outputs in Fig. 2.
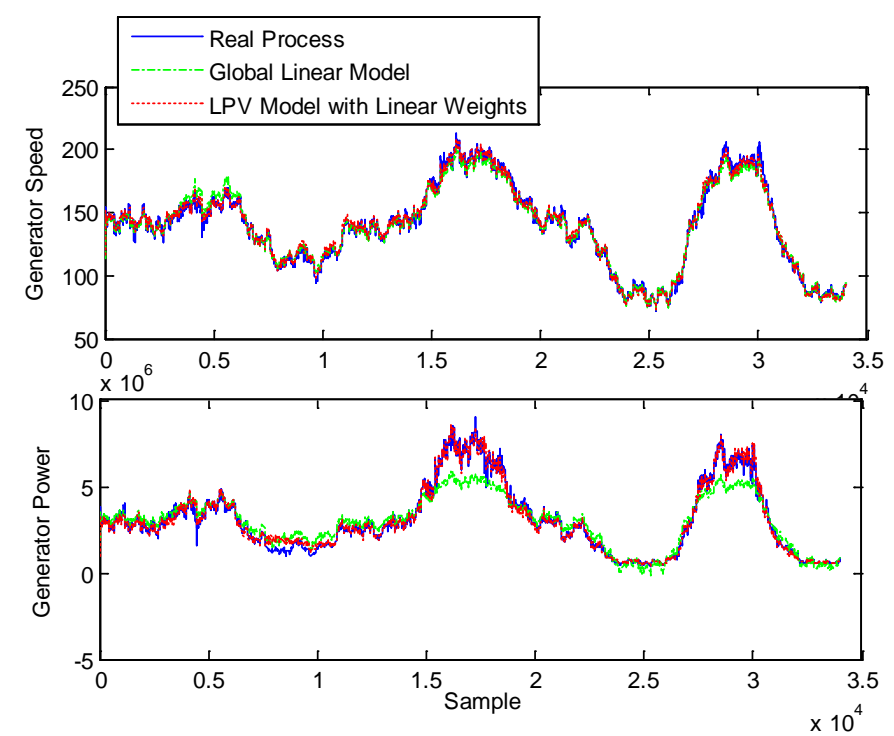

Fig. 2. Outputs of the LPV models with linear weightings

\section{Conclusion}

This paper considers a LPV model structure for wind turbine modeling. The structure is composed by several local linear models with weightings. OE models are selected as local linear models. Linear functions are used as weightings in model identification. Wind speed and pitch angle are selected as scheduling variables. A simulated mechanical wind turbine process is employed. The simulation results show that the LPV models can yield good result with respect to simulation outputs.

\section{Acknowledgements}

This work was supported by the National Natural Science Foundation of China (61174161, 61304141, 61375077); the specialized Research Fund for the Doctoral Program of Higher Education of China (20130121130004); and the Fundamental Research Funds for the Central Universities in China (Xiamen University: No. CBX2014038).

\section{References}

[1] E. A. Bossanyi: Wind Energy. Vol. 6, no. 3 (2003), p. 229.

[2] P. F. Odgaard, J. Stoustrup, and M. Kinnaert: On. Vol. 21, no. 4 (2013), p. 1168.

[3] Y. C. Zhu and Z. H. Xu: Int. Fed. Autom. Control (2008) p. 6.

[4] G. Ji, J. Huang, K. Zhang, Y. Zhu, W. Lin, T. Ji, S. Zhou, and B. Yao: Knowl.-Based Syst. Vol. 45 (2013), p. 62.

[5] J. Huang, G. Ji, Y. Zhu, and P. van den Bosch: J. Process Control. Vol. 22, no. 7 (2012), p. 1198.

[6] A. Junyent-Ferré, O. Gomis-Bellmunt, A. Sumper, M. Sala, and M. Mata: Simul. Model. Pract. Theory. Vol. 18, no. 9 (2010), p. 1365.

[7] P. F. Odgaard, J. Stoustrup, and M. Kinnaert: Proceedings of the 7th IFAC Symposium on Fault Detection, Supervision and Safety of Technical Processes. Vol. 1 (2009), p. 155. 